\title{
SEISMIC SOLAR MODELS AND THE NEUTRINO PROBLEM
}

\author{
M. TAKATA AND H. SHIBAHASHI \\ Department of Astronomy, School of Science, University of Tokyo \\ Bunkyo-ku, Tokyo 113-0032, Japan
}

\begin{abstract}
We determine the structure of the solar radiative zone with the imposition of the sound speed profile and the depth of the convection zone obtained from helioseismic analysis. We discuss the neutrino fluxes and capture rates using the resultant seismic solar model. We find that the seismic solar model cannot resolve the solar neutrino problem. The hydrogen and helium profiles of the Sun are obtained as a part of the solutions. We find that hydrogen is reduced in the core as expected in the theory of stellar evolution.
\end{abstract}

\section{Introduction}

The solar neutrino problem is a long-standing problem in astrophysics and physics. The detected solar neutrino fluxes are substantially less than the theoretical predictions. This discrepancy implies either (i) the physics of neutrinos is not fully understood, or (ii) something is wrong with the solar modelling. The theoretical predictions have been made by using the "standard" solar models. However, these models have the following problems. (1) They are not completely consistent with the results of helioseismology. (2) They are constructed with the assumptions concerning the history of the Sun, which may not be fully justified. (3) They are constructed by adjusting efficiency of the convective energy transport as a free parameter, which is not determined uniquely in the current theory of convection. In this paper, we depart from the standard construction of a solar model and construct a solar model by using as many of the experimentally well measured quantities, including the seismically determined sound speed profile (Takata and Shibahashi, 1997), as possible. We compute the neutrino fluxes based on the resultant solar model and compare them with the observations. First, we determine the sound speed profile of the Sun from the observations of solar oscillations. We then construct a solar model by solving the basic equations governing the stellar structure with the imposition of the determined sound-speed profile and with a constraint of the depth of the convection zone, which is also determined from the sound-speed profile. This method has the following advantages. (1) The model is consistent with helioseismology. (2) The model is a snapshot model of the present-day Sun so that we need few assumptions about the past history of the Sun. (3) We do not care about the treatment of convection since we treat only the radiative core by setting the outer boundary at the base of the convection zone. 

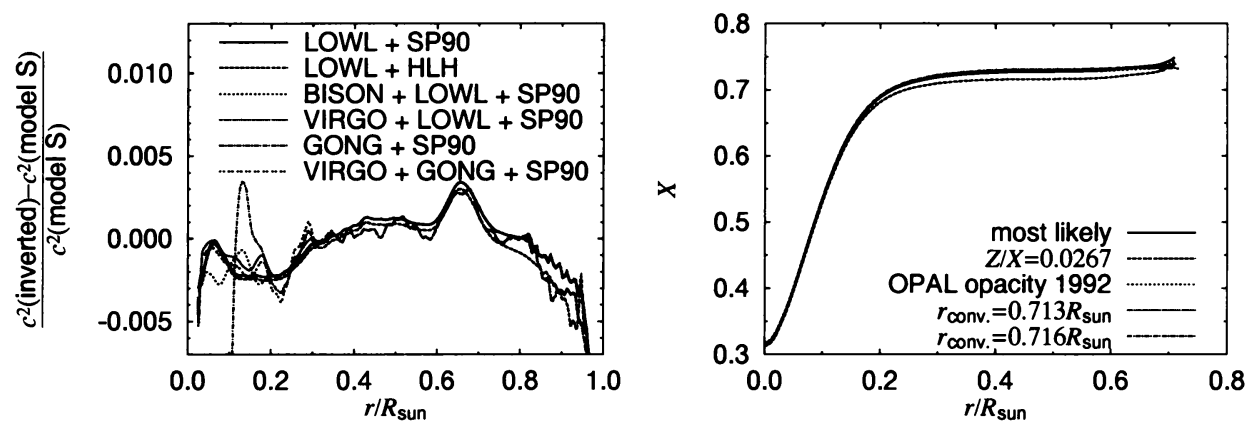

Figure 1. Inverted squared sound speed (left) and hydrogen (right) profiles.

\section{Making a Seismic Solar Model}

We perform an asymptotic inversion to determine the sound speed profile using the data from LOWL (Tomczyk et al., 1995), GONG (Hill et al., 1996), VIRGO on SOHO (Fröhlich et al., 1997), BISON (Elsworth et al., 1994), HLH (Bachmann et al., 1995), and the observation carried out in 1990 at the South Pole (Jefferies et al., 1995). To eliminate the possibility of spurious results, we calibrate the results of the inversion of the observed frequencies using the inverted results of the theoretical frequencies of the same modes of a solar model. Both the statistical error and the systematic error are as low as $0.1 \%$ for $r / R_{\odot}>0.3$ and $0.3 \%$ for $r / R_{\odot}<0.3$. The sound speed profiles determined from various combinations of these data are shown in the left panel of figure 1 . The ordinate is the relative difference between the determined squared sound speed and that of the model S of Christensen-Dalsgaard et al. (1996). Almost all the curves are consistent each other except that the results deduced from the GONG + SP90 (South Pole 1990) data show peculiar behaviour near the center. A conspicuous feature is a hump near $r \simeq 0.65 R_{\odot}$. The general feature of the present results are almost consistent with the recent inversions made by other groups (e.g. Basu et al. 1996, Kosovichev et al. 1997). The location of the base of the convection zone is determined from the fact that the function $W \equiv r^{2} /\left(G M_{\odot}\right) d c^{2} / d r$ becomes almost constant in the convection zone. The present results are consistent with Gough et al. (1996)'s estimate, $r_{\text {conv }}=0.709 R_{\odot}$. Once the sound speed profile is determined, we solve the basic equations governing the stellar structure with the imposition of the determined sound speed profile. The hydrogen and helium profiles are obtained as a part of solutions as well as other thermodynamical quantities. We assume, as the first step, that $Z$ is constant through the whole Sun. Note that recent evolutionary solar models including metal diffusion (e.g. Bahcall and Pinsonneault 1995, ChristensenDalsgaard et al. 1996) are found to be more consistent with the sound speed profile obtained by helioseismology than those without metal diffusion. We will try inhomogeneous $Z$ profiles later. The inner boundary conditions are $L_{r}=M_{r}=0$ at $r=0$. The outer boundary conditions are set at the base of the convection zone, and they are (1) $L_{r}=L_{\odot}$ and (2) $\nabla_{\mathrm{rad}}=\nabla_{\mathrm{ad}}$. The latter means that the neutral stability against convection holds at the base of the convection zone. We also impose that the relative abundance of heavy elements to hydrogen is equal to the photospheric value, 
TABLE 1. Neutrino capture rates and fluxes.

\begin{tabular}{|c|c|c|}
\hline & Seismic Model & Experiments \\
\hline $\mathrm{Cl}[\mathrm{SNU}]$ & $9.4_{-1.9}^{+1.6}$ & $2.28 \pm 0.23^{* 1}$ \\
\hline $\mathrm{Ga}[\mathrm{SNU}]$ & $137_{-8}^{+7}$ & $\begin{array}{l}\left.69.7 \pm 6.7 \text { (stat. })_{-4.5}^{+3.9} \text { (syst. }\right)^{* 2} \\
69 \pm 10 \text { (stat.) } \pm 6 \text { (syst. })^{* 3}\end{array}$ \\
\hline${ }^{8} \mathrm{~B}\left[10^{6} \mathrm{~cm}^{-2} \mathrm{~s}^{-1}\right]$ & $6.7_{-1.5}^{+1.3}$ & $\begin{array}{l}2.80 \pm 0.19 \text { (stat.) } \pm 0.33 \text { (syst.) })^{* 4} \\
2.51_{-0.13}^{+0.14} \text { (stat.) } \pm 0.18 \text { (syst.) }{ }^{* 5}\end{array}$ \\
\hline
\end{tabular}

References. $-{ }^{* 1}$ Homestake (Davis, 1993), ${ }^{* 2}$ GALLEX (Hampel et al., 1996), ${ }^{* 3}$ SAGE (Abdurashitov et al., 1994), ${ }^{* 4}$ Kamiokande (Fukuda et al., 1996), ${ }^{* 5}$ Super-Kamiokande (Totsuka, 1997)

$Z / X=0.0245$ (Grevesse and Noels, 1993), since the matter in the convection zone is homogeneous due to mixing. We adopt the OPAL equation of state (Rogers et al., 1996), the OPAL opacity (Iglesias and Rogers, 1996), and the nuclear reaction rates compiled by Bahcall and Pinsonneault (1995).

\section{Results and Discussion}

The hydrogen profile of the most likely seismic solar model is shown by the thick solid curve in the right panel of figure 1 . To see the influence of the input physics upon the results, we vary the value of $Z / X$, the depth of the convection zone, and the opacity. The results are also shown in the right panel of figure 1 . The central hydrogen is really reduced in all cases as expected in the standard theory of stellar evolution. We estimate the age of the present-day Sun $\left(t_{\odot}\right)$ from the amount of hydrogen which was converted to helium. Assuming that the average solar luminosity during evolution is $0.85 L_{\odot}$, we obtain $t_{\odot} \sim 5.5 \times 10^{9} \mathrm{yr}$. This is the helioseismic determination of the solar age and is independent of the conventional meteoritic determination. We also obtain the helium profile. The estimated surface helium abundance $\left(Y_{\mathrm{S}}\right)$ is between 0.23 and 0.25 . This value is consistent with the other helioseismic results determined from the variation in the adiabatic exponent in the HeII ionization zone (Degl'Innocenti et al., 1997). Though the value itself is not very accurate, it should be emphasized that the present method is a new one of determining $Y_{\mathrm{S}}$. Table 1 lists the neutrino capture rates and fluxes of the seismic solar model and those of experiments. We estimated the errors in the theoretical prediction by taking account of the uncertainties of various input parameters including the sound speed, nuclear reaction rates, opacity, chemical composition, screening effects, position of the base of the convection zone and the systematic errors of inversion process. As seen in table 1, the theoretical values based on the seismic solar model are still significantly larger than the observed ones. It should be stressed that we are free from the standard evolutionary processes and have constructed a snapshot model of the present Sun with fewer assumptions. Nevertheless, the large discrepancy between theory and experiments still exists. Hence, we conclude that the astrophysical solution to the solar neutrino problem is unlikely.

There is another kind of inversion technique called the non-asymptotic inversion, 
which is based on the variational principle. Some of the data used in the present paper have also been inverted by the non-asymptotic method (e.g., Basu et al. 1996, Gough et al. 1996). An advantage of non-asymptotic inversion methods is that we get directly one more physical quantity such as $\rho$ in addition to the sound speed profile. The overall features of the non-asymptotically inverted sound speed profile are similar to the present results. However, the central density obtained in the present study is higher by several percent than the result of the non-asymptotic inversion. The cause of this difference is not yet clear. One possibility is the difference in the assumption on the $Z$-profile. As a numerical experiment, we depart from the assumption of constant $Z$ and increase $Z$ in the radiative core while we keep $Z / X$ constant at the base of the convection zone. We find out how much we need to modify $Z$ so that the inverted density near the center in our method matches with the non-asymptotically inverted density. The result is $Z_{\mathrm{c}} \simeq 0.04$. This value is much larger than the standard values, and we think that the difference only in the $Z$-profile is unlikely to be the cause of inconsistency in the inverted density profiles. The non-asymptotically inverted density profile should be independent of the input physics such as the equation of state, opacity and nuclear reaction rates. On the other hand, the density profile obtained in our method is dependent on them. The apparent discrepancy of the density profiles may imply that something is wrong in the input physics.

\section{References}

Abdurashitov, J. N. et al. : 1994, Phys. Lett. B 328, 234.

Bachmann, K. T., Duvall, T. L., Jr., Harvey, J. W., and Hill, F.: 1995, Astrophys. J. 443, 837.

Bahcall, J. N. and Pinsonneault, M. H.: 1995, Rev. Mod. Phys. 67, 781.

Basu, S., Christensen-Dalsgaard, J., Schou, J., Thompson, M. J., and Tomczyk, S.: 1996, Bull. Astr. Soc. India 24, 147.

Christensen-Dalsgaard, J. et al.: 1996, Science 272, 1286.

Davis, R., Jr.: 1993, in Y. Suzuki and K. Nakamura (eds.), Frontiers of Neutrino Astrophysics, Universal Academy Press, Tokyo, p. 47.

Degl'Innocenti, S., Dziembowski, W., Fiorentini, G., and Ricci, B.: 1997, Astroparticle Physics 7, 77.

Elsworth, Y., Howe, R., Isaak, G. R., McLeod, C. P., Miller, B. A., New, R., Speake, C. C., and Wheeler, S. J.:1994, Astrophys. J. 434, 801.

Fröhlich, C. et al. : 1997, Solar Phys. 170, 1.

Fukuda, Y. et al.: 1996, Phys. Rev. Lett. 77, 1683.

Gough, D. O. et al. : 1996, Science 272, 1296.

Grevesse, N. and Noels, A.: 1993, in N. Prantzos, E. Vangioni-Flam and M. Cassé (eds.), Origin and Evolution of the Elements, Cambridge University Press, Cambridge, p. 15.

Hampel, W. et al. : 1996, Phys. Lett. B 388, 384.

Hill, F. et al. : 1996, Science 272, 1292.

Iglesias, C. A. and Rogers, F. J.: 1996, Astrophys. J. 464, 943.

Jefferies, S. M., Duvall, T. L., Jr., Harvey, J. W., and Pomerantz, M. A.: 1995, private communication.

Kosovichev, A. G. et al. : 1997, Solar Phys. 170, 43.

Rogers, F. J., Swenson, F. J., and Iglesias, C. A.: 1996, Astrophys. J. 456, 902.

Takata, M. and Shibahashi, H.: 1997, Astrophys. J., submitted.

Tomczyk, S., Streander, K., Card, G., Elmore, D., Hull, H., and Cacciani, A.: 1995, Solar Phys. 159, 1.

Totsuka, Y.: 1997, in A. Olinto, J. Frieman and D. Schramm (eds.), Proc. 18th Texas Symp. on Relativistic Astrophys., World Scientific Press, Singapore, in press. 